\title{
Abrogation of Salmonella and E. coli O157:H7 in Feedlot Cattle Fed a Proprietary Saccharomyces cerevisiae Fermentation Prototype
}

Kristina M Feye ${ }^{1}$, Kristi L Anderson ${ }^{1}$, Mark F Scott ${ }^{2}$, Darin L Henry ${ }^{2}$, Kristy L Dorton ${ }^{2}$, Brandon E Depenbusch ${ }^{3}$ and Steve A Carlson ${ }^{\text {* }}$

${ }^{1}$ Department of Biomedical Sciences, lowa State University College of Veterinary Medicine, Ames, IA 50011, USA

${ }^{2}$ Diamond V, Cedar Rapids, IA 52404, USA

${ }^{3}$ Innovative Livestock Services, Great Bend, KS 67530, USA

\begin{abstract}
Salmonella and E. coli O157: $\mathrm{H} 7$ are insidious problems for the beef industry. Asymptomatic fecal shedding of these pathogens contaminates the hide and carcass. Furthermore, Salmonella are unique in their ability to infiltrate lymph nodes leading to the post-harvest contamination of ground beef. These contaminations yield the two most important food safety hazards associated with the consumption of beef. Herein, we report the anti-Salmonella and anti-E. coli O157: $\mathrm{H} 7$ effects of a novel Saccharomyces cerevisiae fermentation prototype (PRT; NaturSafe ${ }^{\mathrm{TM}}$ ) fed to feedlot cattle. Cattle fed PRT were compared to those fed a combination of monensin, tylosin, and a direct-fed microbial- a standard conventional practice in the U.S. beef industry. In this investigator-blinded study, 1,495 feedlot heifers (300-400 kg) were fed PRT ( $\mathrm{n}=747$ heifers) or the standard industry diet (PC; $\mathrm{n}=748$ heifers) without PRT for 125-146 days prior to slaughter. At the abattoir, fecal swabs were obtained from 400 animals ( $n=200 / g r o u p)$ and subjected to selective culture for enumerating Salmonella and E. coli O157:H7. Additionally, subiliac lymph nodes were obtained from 400 carcasses for enumeration of Salmonella spp. Salmonella isolated from the feces and lymph nodes were subjected to a virulence assay and some antibiotic susceptibility and Salmonella serovar testing. When compared to cattle that received PC, Salmonella fecal shedding, lymph node infiltration, virulence, and antibiotic resistances were significantly decreased in cattle fed PRT. Additionally, PRT-fed cattle had a lower prevalence of certain Salmonella serovars (Newport, Typhimurium, and Dublin) and shed fewer E. coli O157:H7. The decrease in Salmonella virulence was associated with a decreased expression of hilA, a genetic regulator of Salmonella invasion into eukaryotic cells. This study revealed that a proprietary Saccharomyces cerevisiae fermentation prototype inhibits the shedding, lymph node carriage, downstream virulence, and antibiotic resistance of Salmonella residing in cattle beyond the standard conventional practice that includes monensin, tylosin, and a direct-fed microbial.
\end{abstract}

Keywords: Salmonella; Cattle; Saccharomyces cerevisiae; Fermentation product

\section{Introduction}

Bovine-associated Salmonella are capable of causing clinical disease, but these Salmonella can also asymptomatically reside in the intestinal tract and lymph nodes of cattle. Both of these reservoirs create food safety hazards, especially the latter situation where lymph nodes serve as a protective conduit for Salmonella passage into ground beef [1]. The intestines also serve as a reservoir for $E$. coli $\mathrm{O} 157: \mathrm{H} 7$, which is nonpathogenic in cattle but highly pathogenic in humans [2].

Intestinal Salmonella and E. coli O157:H7 lead to surface contamination of beef, which can be mitigated by a number of different strategies at the abattoir [3]. Lymph node infiltration, on the other hand, is problematic given the number of lymph nodes unavoidably co-harvested with ground beef and the covertness of the Salmonella within the lymph nodes [1]. Concerns with Salmonella in the lymph nodes underscore the need for improving Salmonella prophylaxis either in the preharvest or post-harvest setting. Recent studies have demonstrated that soluble components present in Saccharomyces cerevisiae fermentation products (SCFP; SmartCare and Original XPC ${ }^{\mathrm{mx}}$, Diamond V) eliminate Salmonella from the gastrointestinal tract of experimentally-infected calves [4]. Specifically, SCFP (SmartCare and Original XPC) significantly reduced the intestinal burden and pathogenic effects of Salmonella in calves [4]. Because of these benefits, the anti-Salmonella effects of SCFP were examined in feedlot cattle naturally infected with Salmonella. The aims of this study were to determine a broad range of effects of a proprietary S. cerevisiae fermentation prototype (PRT; NaturSafe ${ }^{\mathrm{TM}}$ ) on Salmonella when fed to finishing cattle. Specifically fecal shedding, lymph node infiltration, downstream virulence, antibiotic resistance, and the prevalence of virulent Salmonella serotypes with a broad host range were determined. The virulence aspect was examined, in part, because SCFP (EpiCor, Embria Health Sciences) can increase intestinal butyrate production [5], and this short-chain fatty acid has been shown to inhibit Salmonella virulence mechanisms in vitro [6]. The antibiotic resistances were examined since SCFP (Original XPC) can modulate the microbiome $[7,8]$, which can alter the antibiograms of resident bacteria [9]. Additionally, we examined the effects of the SCFP on the shedding of $E$. coli $\mathrm{O} 157: \mathrm{H} 7$ since this microbe shares mammalian cell adhesion strategies with Salmonella [10]. Comparisons were made to cattle fed a combination of monensin, tylosin, and a direct-fed microbial, which is a standard conventional practice in the U.S. beef industry.

\section{Materials and Methods}

\section{Animal care and study design}

Heifers ( $\mathrm{n}=1,495 ; 300-400 \mathrm{~kg})$ were obtained from two sale barns $(n=438)$ and one backgrounding facility $(n=1,057)$ in Kansas in May of 2015. Cattle were shipped to a commercial feedlot in Central Kansas and were provided water and hay ad libitum. On day 1 post-arrival,

*Corresponding author: Steve A Carlson, Department of Biomedical Sciences, lowa State University College of Veterinary Medicine, Ames, IA 50011, USA, Tel: 515-294-0912; Fax: 515-294-2315; E-mail: stevec@iastate.edu

Received May 25, 2016; Accepted June 15, 2016; Published June 20, 2016

Citation: Feye KM, Anderson KL, Scott MF, Henry DL, Dorton KL, et al. (2016) Abrogation of Salmonella and E. coli O157:H7 in Feedlot Cattle Fed a Proprietary Saccharomyces cerevisiae Fermentation Prototype. J Vet Sci Technol 7: 350 doi:10.4172/2157-7579.1000350

Copyright: ( 2016 Feye KM, et al. This is an open-access article distributed under the terms of the Creative Commons Attribution License, which permits unrestricted use, distribution, and reproduction in any medium, provided the original author and source are credited. 
heifers were individually weighed, identified, implanted, and vaccinated using standard procedures at the feedlot. Heifers were then randomly assigned into pens in groups of five until each pen reached its optimal capacity ( 75 animals) based on bunk space and the area of the pen (14.4 inches of bunk space and 231 square feet of pen space per animal).

Two adjacent pens were designated as a single block and 10 blocks were created within the feedlot. Pens of heifers in each block were provided either a diet that contained a combination of standard industry technologies (PC), including monensin (Rumensin, Elanco Animal Health, $300 \mathrm{mg} / \mathrm{head} /$ day), tylosin (Tylovet, Huvepharma, 90 $\mathrm{mg} /$ head/day) and a direct-fed microbial (Bovamine Defend, Nutrition Physiology Company, $50 \mathrm{mg} /$ head/day); or a diet containing $18 \mathrm{gm} /$ head/day of a $S$. cerevisiae fermentation prototype (PRT; NaturSafe ${ }^{\mathrm{TM}}$, Diamond V) without monensin, tylosin, or the direct-fed microbial. Heifers received three step-up diets prior to their final finishing diet (Table 1). All treatment feed additives were stored under manufacturerrecommend conditions and added to the final ration using a microingredient weight machine (Micro Beef Technologies, Amarillo, TX).

During the study, heifers were monitored for illness and treated as per recommendations by a veterinarian. Heifers that responded to treatment were returned to the study while non-responders were removed from the study. Morbidities and mortalities were indistinct between the two groups (data not shown).

At the conclusion of the study, heifers were shipped 145 miles to a commercial abattoir on two separate dates that were three weeks apart. These shipping dates corresponded to 125 and 146 days on study for the first and second groups, respectively. An equal number of pens per treatment group were shipped on each date ( $n=5$ per treatment). Shipments and carcass processing occurred on a pen-by-pen basis.

\section{Sample collection at the abattoir}

Individual animal numbers were not carried through the entire harvest process. Only the first and last animal within a pen was tagged with a lot identification tag that corresponded back to the pen number. Since individual identification was not maintained, it was not possible to keep track of which animals were sampled. As a result, lymph node and fecal samples may or may not have been collected from the same animals.

Fecal swabs were collected on the rail from 20 animals per pen (replicate). Samples were collected from every third or fourth animal

\begin{tabular}{|c|c|c|c|c|}
\hline Ingredient, \% of dry matter & Starter & Ration 2 & Ration 3 & Finisher \\
\hline Steam-flaked corn & 30.2 & 45.8 & 58.6 & 66.1 \\
\hline Wet distiller's grain & 22.2 & 19.5 & 18.0 & 18.0 \\
\hline Alfalfa hay & 38.0 & 25.0 & 13.0 & - \\
\hline Corn stalks & - & - & - & 4.0 \\
\hline Corn silage & 7.0 & 7.0 & 5.0 & 4.0 \\
\hline Tallow 1,2 & - & - & 1.5 & 2.9 \\
\hline Supplement & 2.6 & 2.7 & 3.9 & 5.0 \\
\hline
\end{tabular}

Control rations were formulated to provide $300 \mathrm{mg}$ of monensin (Elanco Animal Health, Greenfield, IN), $90 \mathrm{mg}$ of tylosin (Zoetis Animal Health, Florham, NJ), 0.5 $\mathrm{mg}$ of melengestrol acetate (Zoetis Animal Health), and $50 \mathrm{gm}$ Bovamine Defend (Nutrition Physiology Company, Overland Park, KS) per heifer daily throughout the study, and $250 \mathrm{mg}$ of ractopamine hydrochloride (Zoetis Animal Health) per heifer daily during the last 28 days on feed.

Rations containing PRT were formulated to provide $18 \mathrm{gm}$ of a Saccharomyces cerevisiae fermentation prototype ((NaturSafe ${ }^{\mathrm{TM}}$; Diamond V, Cedar Rapids, IA) and $0.5 \mathrm{mg}$ of melengestrol acetate (Zoetis Animal Health) per heifer daily throughout the study, and $250 \mathrm{mg}$ of ractopamine hydrochloride (Zoetis Animal Health) per heifer daily during the last 28 days on feed.

Table 1: Composition of diets. within a replicate. Fecal samples were collected using a 3M-sponge stick pre-saturated with buffered peptone water. Sponge sticks were inserted into the rectum (recto-anal junction) to collect the sample. After the sample was collected, the sponge was placed into a pre-labeled bag containing buffered peptone water. The bag was closed and placed into a cooler.

Subiliac lymph nodes and the surrounding tissue were collected postevisceration. Sample collection began with the first carcass in each replicate and continued with every third or fourth carcass within that replicate. Lymph nodes were placed into pre-labeled Whirlpak bags. The bags were closed and placed in a cooler. Fecal swabs and lymph node samples were then immediately shipped on ice to Iowa State University College of Veterinary Medicine (Ames, Iowa, USA) for microbiological analyses.

\section{Assessment of Salmonella fecal load and lymph node infiltration}

Salmonella spp. were enumerated from every fecal swab sample and lymph node collected (20 per pen; 200 per treatment) using selective agar (XLD) methods described by Brewer et al. [4]. Approximately $0.3 \mathrm{gm}$ of feces or lymph node were collected on a sterile cotton swab and then aseptically transferred into $10 \mathrm{~mL}$ Lennox broth and an aliquot of the broth was immediately plated on XLD agar, incubated overnight at $37^{\circ} \mathrm{C}$, and subjected to enumeration by manual counting of black-centered colonies the next day. Load was then determined as (colonies recovered) $\times$ (the dilution factor) / gm of feces or lymph node. Prevalence was calculated as percent of heifers harboring any Salmonella and was compiled across pens within a treatment group.

\section{Assessment of $E$. coli O157:H7 in the Feces}

E. coli $\mathrm{O} 157: \mathrm{H} 7$ was enumerated in 100 of the swab samples (five per pen; 50 swab samples per treatment) using selective media (Sorbitol-MacConkey agar) and a PCR targeting E. coli O157:H7 virulence genes as per Sharma and Casey [11]. Approximately $0.3 \mathrm{gm}$ of feces were transferred into enrichment broth [11] and an aliquot of the broth was plated on sorbitol-MacConkey agar, incubated overnight at $37^{\circ} \mathrm{C}$, and subjected to enumeration by manual counting of nonfermenting colonies the next day. From each pen-specific set of agar plates, 96 colonies were selected and subjected to the PCR targeting $E$. coli O157:H7 virulence genes. Load was then determined as (colonies recovered $\times$ the dilution factor $\times$ the percent of colonies yielding an $E$. coli O157H7-specific amplicon within the pen) / gm of feces. Prevalence was calculated as percent of heifers harboring any E. coli O157:H7 within a pen, and was compiled across pens within a treatment group.

\section{Assessment of tissue culture invasiveness by Salmonella recovered from cattle}

Approximately $50 \%$ of the recovered Salmonella were subjected to a standard antibiotic protection-based tissue culture invasion assay [12] adapted for use with both antibiotic-susceptible and antibiotic-resistant Salmonella [13]. Colonies were collected en masse, on a pen-by-pen basis, into nutrient broth and then immediately incubated for $1 \mathrm{hr}$ with $\mathrm{HEp}-2$ tissue culture cells at $37^{\circ} \mathrm{C}$. Bacteria were recovered from inside tissue culture cells via cell lysis, incubated on XLD agar overnight at $37^{\circ} \mathrm{C}$, and enumerated the next day. Percent invasion was determined as (number of black-centered colonies recovered from inside cells / number of colonies added to cells) $\times 100$.

\section{Assessment of virulence gene expression in Salmonella isolated from cattle}

In order to correlate the virulence of Salmonella recovered from cattle with gene expression events in the pathogen, approximately 
$10 \%$ of the recovered Salmonella isolates were subjected to an assay that quantitates the expression of hilA (a key regulator of Salmonella invasion genes; [14]). RNA was extracted from the isolates that were collected en masse on a pen-by-pen basis, and then subjected to a semiquantitative RT-PCR targeting the hilA transcript [15]

\section{Assessment of antibiotic resistance in Salmonella recovered from cattle}

Approximately $20 \%$ of the recovered Salmonella were individually subjected to micro-broth assays with individual antibiotics (florfenicol, ceftiofur, and enrofloxacin) at breakpoint concentrations [16]. Colonies that grew in the breakpoint concentrations were deemed to be resistant. Percent resistant were then determined as (number of resistant colonies / number of colonies examined) x 100. Data were compared across pens and between groups.

\section{Assessment of the presence of three important Salmonella serotypes in the samples}

Nearly $20 \%$ of the recovered Salmonella were individually subjected to PCR assays that detect the presence of genes related to Dublin [17], Typhimurium [17], and Newport [18] serotypes. Colonies yielding a specific PCR amplicon(s) were deemed to belong to the ascribed serotype. Percent belonging to the serotype were then determined as (number of colonies yielding a specific amplicon / number of colonies examined) $\times 100$. Data were compared across pens and between groups.

\section{Statistical analyses}

Statistical comparisons were made using an analysis of variance with Tukey's ad hoc test for multiple comparisons (GraphPad Prism, Version 6, La Jolla, CA). Significant differences were defined at $P \leq 0.05$.

\section{Results}

\section{Assessment of Salmonella Fecal Shedding in Cattle}

Salmonella can be a potential pathogen source for humans who consume improperly cooked beef. Carcasses can become exposed to Salmonella through fecal contamination during processing. In this study, fecal shedding of Salmonella was evaluated in 200 heifer's postmortem from each treatment group. As shown in Figure 1a, fecal shedding of Salmonella was significantly less $(P<0.05)$ in cattle fed PRT (105 versus $405 \mathrm{CFU} / \mathrm{gm}$ of feces, respectively). The relative prevalence of fecal shedding was significantly less $(P<0.05)$ in heifers fed PRT $(6$ versus $13 \%$, respectively) as per Figure $1 \mathrm{~b}$.

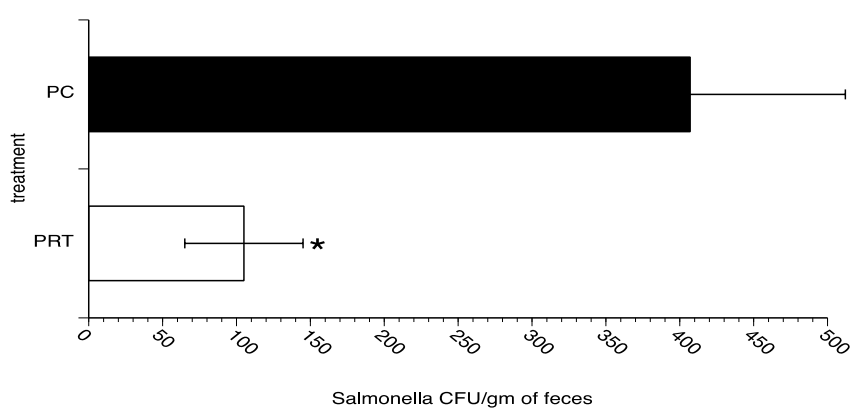

Figure 1a: Salmonella fecal load in heifers fed PRT (NaturSafe ${ }^{\mathrm{TM}}$ ) or PC. The PC diet contained monensin (Elanco Animal Health), tylosin (Huvepharma) and a direct-fed microbial (Nutrition Physiology Company). The PRT diet contained Saccharomyces cerevisiae fermentation prototype ((NaturSafe ${ }^{\mathrm{TM}}$; Diamond V) and no monensin, tylosin, or a direct-fed microbial. Data represent the mean + SEM for measurements on 200 heifers in each group. ${ }^{~} P<0.05$ versus Control.

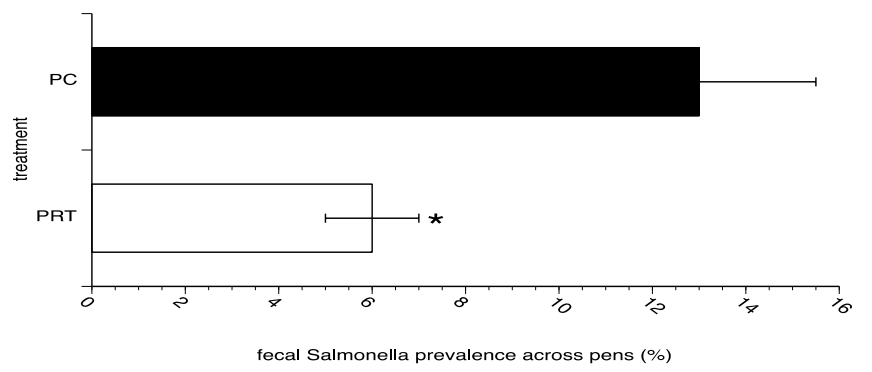

Figure 1b: Prevalence of Salmonella fecal shedding in heifers fed PRT (NaturSafe $^{\mathrm{TM}}$ ) or PC. The PC diet contained monensin (Elanco Animal Health), tylosin (Huvepharma) and a direct-fed microbial (Nutrition Physiology Company). The PRT diet contained Saccharomyces cerevisiae fermentation prototype (NaturSafe ${ }^{\mathrm{TM}}$; Diamond V) and no monensin, tylosin, or a direct-fed microbial. Data represent the mean \pm SEM for the qualitative prevalence $(\%$ of heifers shedding Salmonella) across pens in each group. ${ }^{*} P<0.05$ versus Control.

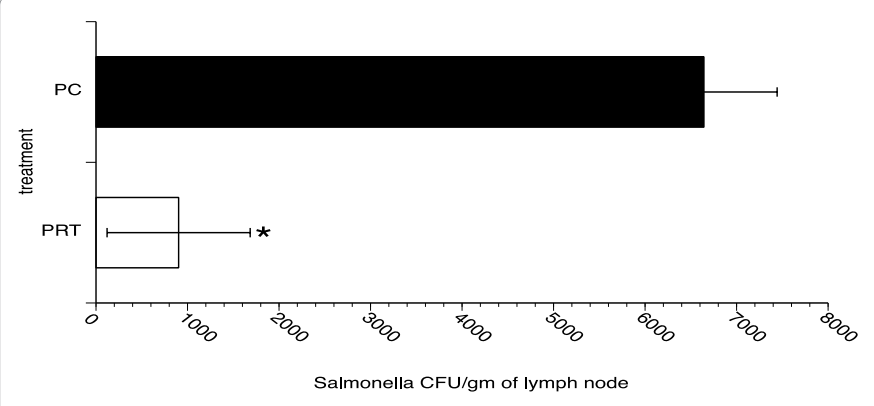

Figure 2a: Assessment of lymph node infiltration by Salmonella in heifers fed PRT (NaturSafe ${ }^{T M}$ ) or PC. The PC diet contained monensin (Elanco Animal Health), tylosin (Huvepharma) and a direct-fed microbial (Nutrition Physiology Company). The PRT diet contained Saccharomyces cerevisiae fermentation prototype (NaturSafe ${ }^{\mathrm{TM}}$; Diamond V) and no monensin, tylosin, or a direct-fed microbial. Data represent the mean + SEM for measurements on 200 heifers in each group. ${ }^{*} P<0.05$ versus Control.

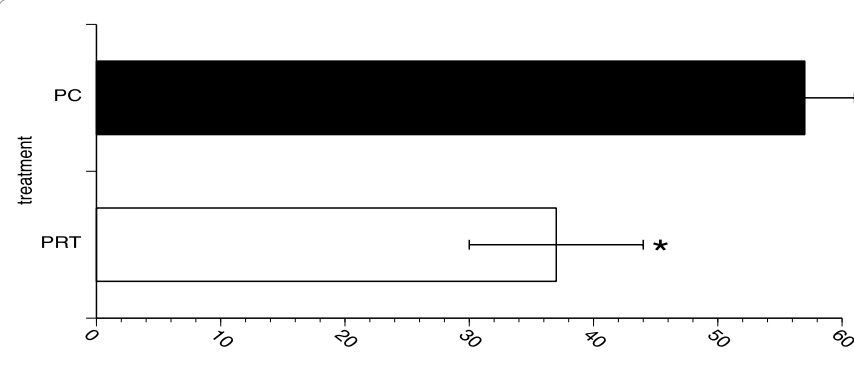

fecal E. coli O157:H7 prevalence across pens (\%)

Figure 2b. Assessment of the prevalence of Salmonella lymph node infiltration in heifers fed PRT (NaturSafe ${ }^{\mathrm{TM}}$ ) or PC. The PC diet contained monensin (Elanco Animal Health), tylosin (Huvepharma) and a direct-fed microbial (Nutrition Physiology Company). The PRT diet contained Saccharomyces cerevisiae fermentation prototype (NaturSafe ${ }^{\mathrm{TM}}$; Diamond $\mathrm{V}$ ) and no monensin, tylosin, or a direct-fed microbial. Data represent the mean + SEM for the qualitative prevalence $(\%$ of heifers shedding Salmonella) across pens in each group. ${ }^{*} P<0.05$ versus Control.

\section{Assessment of lymph node infiltration by Salmonella}

Since Salmonella lymph node carriage can be a source of contamination of ground beef [19], Salmonella load was determined in the subiliac lymph nodes of 200 carcasses from each treatment group. As shown in Figure 2a, lymph node infiltration was significantly less $(P<0.05)$ in carcasses from heifers fed PRT (902 versus 6,642 CFU/gm of lymph node, respectively). The percent of Salmonella-bearing lymph nodes was significantly less $(P<0.05)$ in carcasses from heifers fed PRT (4 versus $14 \%$, respectively) as per Figure $2 \mathrm{~b}$. 


\section{Determination of $E$. coli $0157: \mathrm{H} 7$ in the feces of cattle}

To determine if PRT had an effect on the presence of $E$. coli O157:H7 in the feces of the heifers, fecal samples (100 per treatment group) were quantitatively examined for the presence of this critical foodborne pathogen. As shown in Figure 3a, heifers fed PRT had a statistically lower $(P<0.05)$ E. coli O157:H7 fecal load than heifers fed $\mathrm{PC}$ (52 versus $122 \mathrm{CFU/gm}$ of feces, respectively). Figure $3 \mathrm{~b}$ reveals a decreased prevalence $(P<0.05)$ of $E$. coli O157:H7 in heifers fed PRT when compared to those receiving the PC diet (37 versus $57 \%$, respectively).

\section{Assessment of the virulence of Salmonella recovered from cattle}

In order to compare the virulence of Salmonella recovered from cattle, the isolates were subjected to an assay that predicts the ability of Salmonella to invade gut epithelial cells, which is a hallmark of Salmonella virulence [20]. The effects of PRT on virulence were examined due to the ability of SCFP to increase butyrate in the intestine [5]. Research has shown that butyrate can decrease the Salmonella invasion gene (hilA) expression in vitro [6], which results in the decreased ability of Salmonella to invade cells. In the current study, invasiveness of Salmonella was significantly less $(P<0.05)$ in Salmonella recovered from the feces and lymph nodes of cattle fed PRT (Figure 4). This decrease in

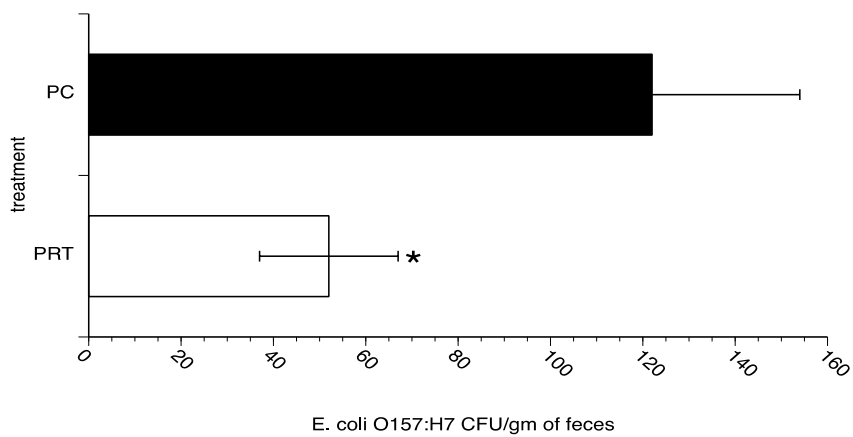

Figure 3a: E. coli O157:H7 fecal load in heifers fed PRT (NaturSafe ${ }^{\mathrm{TM}}$ ) or PC. The $\mathrm{PC}$ diet contained monensin (Elanco Animal Health), tylosin (Huvepharma) and a direct-fed microbial (Nutrition Physiology Company). The PRT diet contained Saccharomyces cerevisiae fermentation prototype (NaturSafe ${ }^{\mathrm{TM}}$; Diamond $\mathrm{V}$ ) and no monensin, tylosin, or a direct-fed microbial. Data represent the mean \pm SEM for measurements on 50 heifers in each group. ${ }^{*} P<0.05$ versus Control.

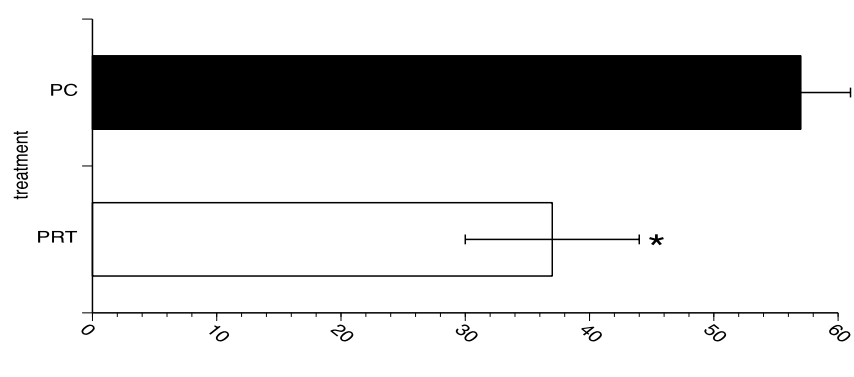

fecal E. coli O157:H7 prevalence across pens (\%)

Figure 3b: Prevalence of $E$. coli O157:H7 fecal shedding in heifers fed PRT (NaturSafe ${ }^{\mathrm{TM}}$ ) or PC. The PC diet contained monensin (Elanco Animal Health), tylosin (Huvepharma) and a direct-fed microbial (Nutrition Physiology Company). The PRT diet contained Saccharomyces cerevisiae fermentation prototype (NaturSafe ${ }^{\mathrm{TM}}$; Diamond $\mathrm{V}$ ) and no monensin, tylosin, or a direct-fed microbial. Data represent the mean \pm SEM for the qualitative prevalence (\% of heifers shedding $E$. coli O157:H7) across pens in each group. ${ }^{*} P<0.05$ versus Control.

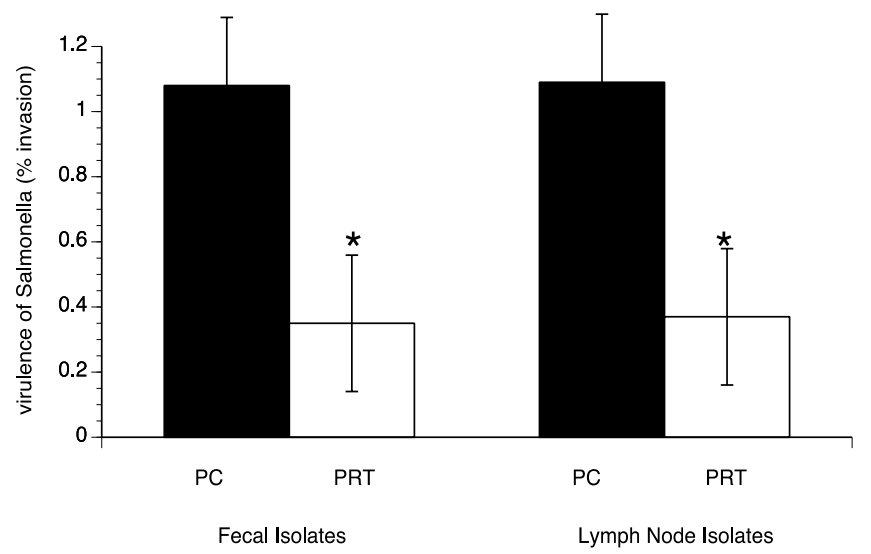

Figure 4: Tissue culture invasiveness of Salmonella recovered from the feces or lymph nodes of heifers fed PRT (NaturSafe ${ }^{\top M}$ ) or PC. The PC diet contained monensin (Elanco Animal Health), tylosin (Huvepharma) and a direct-fed microbial (Nutrition Physiology Company). The PRT diet contained Saccharomyces cerevisiae fermentation prototype (NaturSafe ${ }^{\mathrm{TM}}$; Diamond V) and no monensin, tylosin, or a direct-fed microbial. Data represent the mean \pm SEM for group measurements on Salmonella recovered from 200 heifers in each group. ${ }^{*} P<0.05$ versus Control.

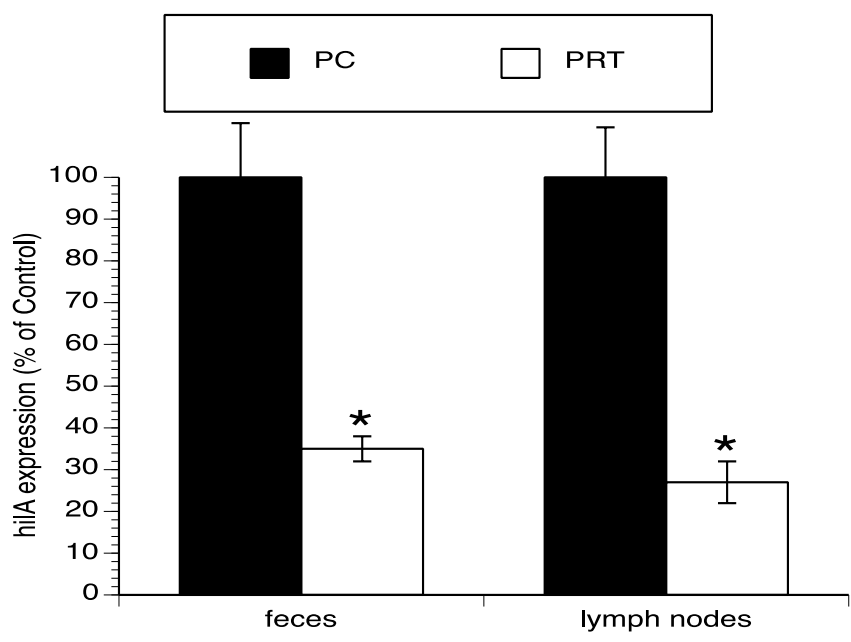

source of the Salmonella

Figure 5: Semi-quantitation of hilA expression of Salmonella recovered from the feces and lymph nodes of heifers fed PRT (NaturSafe ${ }^{\mathrm{TM}}$ ) or PC. The PC diet contained monensin (Elanco Animal Health), tylosin (Huvepharma) and a direct-fed microbial (Nutrition Physiology Company). The PRT diet contained Saccharomyces cerevisiae fermentation prototype (NaturSafe ${ }^{\mathrm{TM}}$; Diamond V) and no monensin, tylosin, or a direct-fed microbial. Data represent the mean \pm SEM for pooled group measurements on Salmonella recovered from feces or lymph nodes. ${ }^{*} P<0.05$ versus Control.

invasiveness coincided with a decrease in the expression of hilA (Figure 5), a major regulator of Salmonella virulence for mammalian hosts [14].

\section{Assessment of the antibiotic resistance of Salmonella recovered from cattle}

The antibiotic resistance of Salmonella in lymph nodes and fecal samples were examined since SCFP can modulate the microbiome $[7,8]$, which can alter the antibiograms of resident bacteria [9]. To assess the possibility that PRT inhibits antibiotic resistant Salmonella or induces the expulsion of antibiotic resistance elements from Salmonella, isolates 
recovered from cattle were subjected to an antibiotic susceptibility assay that utilized three individual antibiotics (ceftiofur, enrofloxacin, and florfenicol). These three antibiotics were chosen given their extended spectra and importance in bovine therapeutics. Additionally, two of the three antibiotics tested (ceftiofur and enrofloxacin) have counterparts important for human therapeutics (ceftriaxone and ciprofloxacin, respectively).

Figure 6 reveals a decrease in the prevalence of resistant $(P<0.05)$ Salmonella recovered from heifers fed PRT for all three antibiotics. This figure represents isolates from both feces and lymph nodes. It is of note that resistance of these antibiotics was, in general, more prevalent in the fecal isolates when compared to the lymph node isolates.

\section{Assessment of the presence of three important Salmonella serotypes in the samples}

Salmonella isolates recovered from the feces or lymph nodes of cattle were subjected to PCR assays targeting three serotypes (Dublin, Newport, and Typhimurium). As shown in Figure 7, the prevalence of two of these serovars was diminished in heifers fed PRT, regardless of the source of the

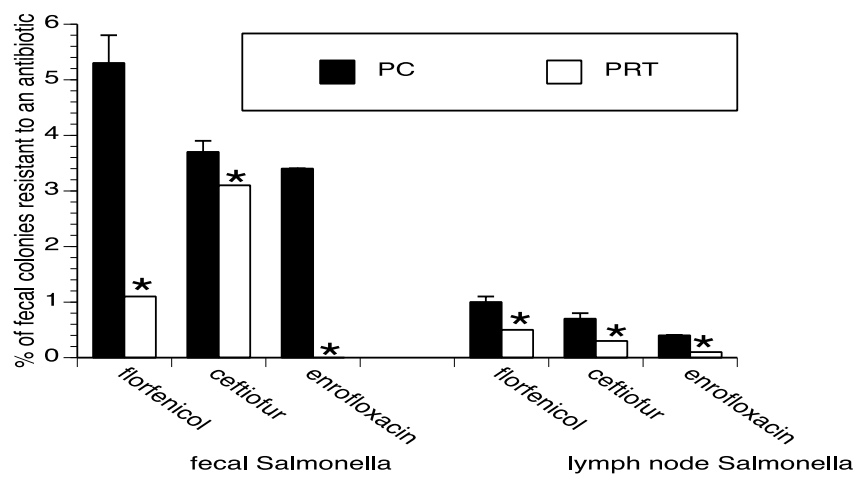

Figure 6: Prevalence of antibiotic resistant Salmonella recovered from the feces and lymph nodes of heifers fed PRT (NaturSafe ${ }^{\mathrm{TM}}$ ) or PC. The PC diet contained monensin (Elanco Animal Health), tylosin (Huvepharma) and a direct-fed microbial (Nutrition Physiology Company). The PRT diet contained Saccharomyces cerevisiae fermentation prototype (NaturSafe ${ }^{\mathrm{TM}}$; Diamond V) and no monensin, tylosin, or a direct-fed microbial. Data represent the mean \pm SEM for pooled group measurements on Salmonella recovered from feces and lymph nodes. ${ }^{*} P<0.05$ versus Control.

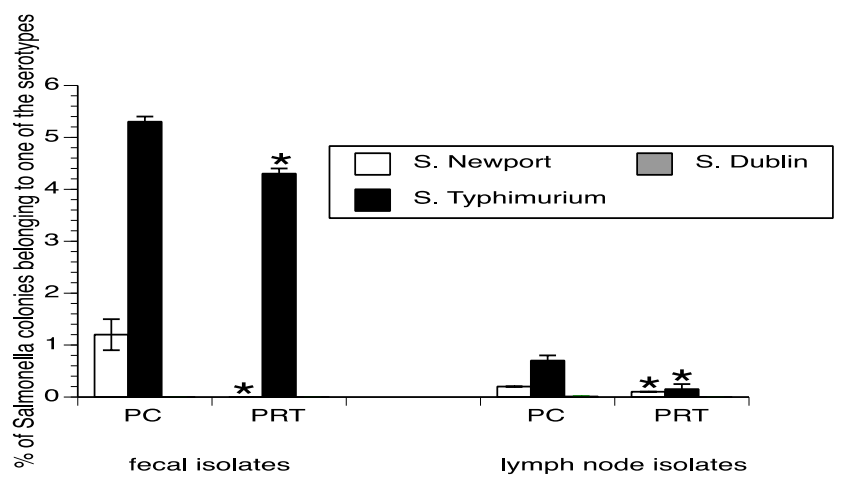

Figure 7: Prevalence of certain Salmonella serotypes recovered from the feces and lymph nodes of heifers fed PRT (NaturSafe ${ }^{\mathrm{TM}}$ ) or PC. The PC diet contained monensin (Elanco Animal Health), tylosin (Huvepharma) and a direct-fed microbial (Nutrition Physiology Company). The PRT diet contained Saccharomyces cerevisiae fermentation prototype (NaturSafe ${ }^{\mathrm{TM}}$; Diamond $\mathrm{V}$ ) and no monensin, tylosin, or a direct-fed microbial. Data represent the mean \pm SEM for group measurements on Salmonella recovered from feces and lymph nodes. ${ }^{*} P<0.05$ versus Control. isolates. No $S$. Dublin were isolated from feces and only one colony of $S$. Dublin was isolated from lymph nodes. Thus, statistical evaluations are not presented for this minor subsection of the study.

\section{Discussion}

Salmonella and E. coli O157:H7 are insidious problems for the beef industry and represent critical food safety hazards. Salmonella and E. coli O157:H7 can be shed in fecal material that can contaminate the carcass during processing. Salmonella is also harbored in the lymph nodes, which can lead to contamination of ground beef. Therefore, identifying mitigation strategies for both pathogens is needed especially considering the covert nature of Salmonella lymph node infiltration [19].

In this study, the anti-Salmonella and anti-E. coli O157:H7 effects of NaturSafe ${ }^{\mathrm{TM}}$ (PRT) were examined and two critical indicators of Salmonella contamination (fecal shedding and lymph node infiltration) were significantly reduced by NaturSafe ${ }^{\mathrm{TM}}$. In this study, heifers fed PC shed a higher number of Salmonella and E. coli O157:H7 and had more Salmonella present in the lymph nodes, which ultimately increases the risk of pathogen transmission to humans that ingest beef. There are currently no other research studies looking at the effects of NaturSafe ${ }^{\mathrm{TM}}$ on pathogens in beef cattle but there are studies examining the effects of other SCFP products on pathogen load. These results described herein are consistent with a previous study in which SCFP (SmartCare and Original XPC) reduced intestinal colonization in pre-ruminant calves [4], which is possibly due to macrophage-associated clearance of the pathogen. In another study, cannulated beef heifers supplemented with SCFP (Original XP', Diamond V) had less E. coli O157:H7 colonizing the recto-anal junction after being challenged with E. coli O157:H7 [21]. Therefore, SCFP products seem to minimize the levels of Salmonella and E. coli $\mathrm{O} 157: \mathrm{H} 7$ in cattle.

Other significant and unique findings in this study were the reduction in virulence and antibiotic resistance in Salmonella recovered from heifers fed PRT. Reduced virulence was detected by diminished tissue culture invasion with a concomitant reduction in the expression of hilA [14]. It is unclear how virulence was mitigated by feeding this SCFP prototype. However, previous studies revealed that SCFP (EpiCor) increases the gastrointestinal production of butyrate [5], a putative repressor of hilA expression [22]. Regardless of the mechanism underlying this effect, the observed magnitude of decreased invasiveness is likely to increase the infectious dose of Salmonella for a human as evidenced by our prior study, in which this level of diminished invasiveness altered the murine $\mathrm{LD}_{50}$ approximately 5-fold [13]

\section{Conclusions}

In summary, NaturSafe ${ }^{\mathrm{TM}}$-fed feedlot heifers were significantly less likely to shed Salmonella and harbor this pathogen in the lymph nodes. The anti-shedding effect of NaturSafe ${ }^{\mathrm{TM}}$ was also observed for E. coli O157:H7. Additionally, NaturSafe ${ }^{\mathrm{TM}}$ reduced the virulence and antibiotic resistance of recovered Salmonella. Ultimately, these beneficial effects will have a marked positive effect on food safety in the beef industry. Future mechanistic studies are warranted to uncover the molecular bases for these effects.

This work was funded by Diamond V Salary support was provided to K.L.A. in exchange for her time devoted to the project, but no other financial compensation was or will be awarded for this study. Thus the authors declare no conflict of interest.

\section{Acknowledgments}

The authors thank Dr. Katelyn Malin for her assistance in sample collection and feedlot personnel for animal husbandry. 
Citation: Feye KM, Anderson KL, Scott MF, Henry DL, Dorton KL, et al. (2016) Abrogation of Salmonella and E. coli O157:H7 in Feedlot Cattle Fed a Proprietary Saccharomyces cerevisiae Fermentation Prototype. J Vet Sci Technol 7: 350. doi:10.4172/2157-7579.1000350

Page 6 of 6

\section{References}

1. Brichta-Harhay DM, Arthur TM, Bosilevac JM, Kalchayanand N, Schmidt JW et al. (2012) Microbiological analysis of bovine lymph nodes for the detection of Salmonella enterica. J Food Prot 75: 854-858.

2. Smith JL, Fratamico PM, Gunther NW $4^{\text {th }}$ (2014) Shiga toxin-producing Escherichia coli. Adv Appl Microbiol 86: 145-197.

3. Soon JM, Chadd SA, Baines RN (2011) Escherichia coli O157:H7 in beef cattle: on farm contamination and pre-slaughter control methods. Anim Health Res Rev 12: 197-211.

4. Brewer MT, Anderson KL, Yoon I, Scott MF, Carlson SA (2014) Amelioration of salmonellosis in pre-weaned dairy calves fed Saccharomyces cerevisiae fermentation products in feed and milk replacer. Vet Microbiol 172: 248-255.

5. Possemiers S, Pinheiro I, Verhelst A, Van den Abbeele P, Maignien L, et al (2013) A dried yeast fermentate selectively modulates both the luminal and mucosal gut microbiota and protects against inflammation, as studied in an integrated in vitro approach. J Agric Food Chem 61: 9380-9392.

6. Gantois I, Ducatelle R, Pasmans F, Haesebrouck F, Hautefort I, et al. (2006) Butyrate specifically down-regulates Salmonella pathogenicity island 1 gene expression. Appl Environ Microbiol 72: 946-949.

7. Mullins CR, Mamedova LK, Carpenter AJ, Ying Y, Allen MS, et al. (2013) Analysis of rumen microbial populations in lactating dairy cattle fed diets varying in carbohydrate profiles and Saccharomyces cerevisiae fermentation product. J Dairy Sci 96: 5872-5881.

8. Price KL, Totty HR, Lee HB, Utt MD, Fitzner GE, et al. (2010) Use of Saccharomyces cerevisiae fermentation product on growth performance and microbiota of weaned pigs during Salmonella infection. J Anim Sci 88: 38963908.

9. Kirkup BC, Riley MA (2004) Antibiotic-mediated antagonism leads to a bacteria game of rock-paper-scissors in vivo. Nature 428: 412-414.

10. Rossez Y, Wolfson EB, Holmes A, Gally DL, Holden NJ (2015) Bacterial flagella: twist and stick, or dodge across the kingdoms. PLoS Pathog 11: e1004483.

11. Sharma VK, Casey TA (2014) Escherichia coli O157:H7 lacking the qseBCencoded quorum-sensing system outcompetes the parental strain in colonization of cattle intestines. Appl Environ Microbiol 80: 1882-1892.
12. Giannella RA, Washington O, Gemski P, Formal SB (1973) Invasion of HeLa cells by Salmonella typhimurium: a model for study of invasiveness of Salmonella. J Infect Dis 128: 69-75.

13. Carlson SA, Browning M, Ferris KE, Jones BD (2000) Identification of diminished tissue culture invasiveness among multiple antibiotic resistant Salmonella typhimurium DT104. Microb Pathog 28: 37-44.

14. Bajaj V, Hwang C, Lee CA (1995) hilA is a novel ompR/toxR family member that activates the expression of Salmonella typhimurium invasion genes. Mo Microbiol 18: 715-727.

15. Carlson SA, Sharma VK, McCuddin ZP, Rasmussen MA, Franklin SK (2007) Involvement of a Salmonella genomic island 1 gene in the rumen protozoanmediated enhancement of invasion for multiple-antibiotic-resistant Salmonella enterica serovar Typhimurium. Infect Immun 75: 792-800.

16. Clinical and Laboratory Standards Institute (2015) Performance standards fo antimicrobial disk and dilution susceptibility tests. Volume 29. 2nd edn, Wayne PA, USA.

17. Akiba M, Kusumoto M, Iwata T (2011) Rapid identification of Salmonella enterica serovars, Typhimurium, Choleraesuis, Infantis, Hadar, Enteritidis, Dublin and Gallinarum, by multiplex PCR. J Microbiol Methods 85: 9-15

18. Cao G, Meng J, Strain E, Stones R, Pettengill J, et al. (2013) Phylogenetics and differentiation of Salmonella Newport lineages by whole genome sequencing. PLoS One 8: e55687.

19. Brichta-Harhay DM, Guerini MN, Arthur TM, Bosilevac JM, Kalchayanand N et al. (2008) Salmonella and Escherichia coli O157:H7 contamination on hides and carcasses of cull cattle presented for slaughter in the United States: an evaluation of prevalence and bacterial loads by immunomagnetic separation and direct plating methods. Appl Environ Microbiol 74: 6289-6297.

20. Millemann $Y$ (1998) Pathogenic power of Salmonellae: virulence factors and study models. Vet Res 29: 385-407.

21. Liou L, Sheng H, Ferens W, Schneider C Hristov A et al. (2009) Reduced Carriage of Escherichia coli O157:H7 in Cattle Fed Yeast Culture Supplement. The Professional Animal Scientist 25: 553-558.

22. Durant JA, Corrier DE, Ricke SC (2000) Short-chain volatile fatty acids modulate the expression of the hilA and invF genes of Salmonella typhimurium. J Food Prot 63: 573-578. 